P. Johannes Helm, Trond Reppen, and Paul Heggelund. A setup for combined multiphoton laser scanning microscopic and multi-electrode patch clamp experiments on brain slices.

Proceedings of SPIE, Vol. 7183, 2009

(C) Copyright 2009 Society of Photo-Optical Instrumentation Engineers. One print or electronic copy may be made for personal use only. Systematic reproduction and distribution, duplication of any material in this paper for a fee or for commercial purposes, or modification of the content of the paper are prohibited.

http://dx.doi.org/10.1117/12.810556

Access to the published version may require journal subscription.

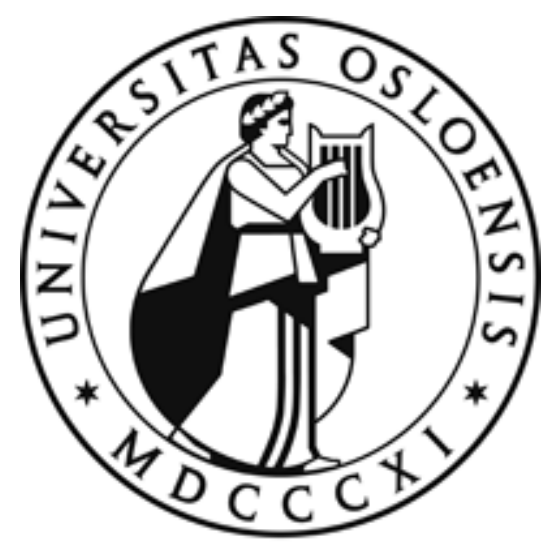




\title{
A setup for combined multi photon laser scanning microscopic and multi electrode patch clamp experiments on brain slices
}

\author{
P. Johannes Helm ${ }^{\mathrm{a}, \ddagger}$, Trond Reppen ${ }^{\mathrm{b}}$, and Paul Heggelund ${ }^{\mathrm{c}}$ \\ ${ }^{\mathrm{a} C e n t r e}$ for Molecular Biology and Neuroscience - University of Oslo, Institute of Basic Medical \\ Sciences, Dept. of Anatomy, P.O. Box 1105 - Blindern, NO-0317 Oslo, Norway \\ ${ }^{b}$ University of Oslo, Institute of Basic Medical Sciences, Laboratory of Electronics, P.O. Box 1104 - \\ Blindern, NO-0317 Oslo, Norway \\ ${ }^{\mathrm{C}}$ University of Oslo, Institute of Basic Medical Sciences, Dept. of Physiology, P.O. Box 1103 - \\ Blindern, NO-0317 Oslo, Norway
}

\begin{abstract}
Multi Photon Laser Scanning Microscopy (MPLSM) appears today as one of the most powerful experimental tools in cellular neurophysiology, notably in studies of the functional dynamics of signal processing in single neurons. Simultaneous recording of fluorescence signals at high spatial and temporal resolution and electric signals by means of multi electrode patch clamp techniques have provided new paths for the systematic investigation of neuronal mechanisms. In particular, this approach has opened for direct studies of dendritic signal processing in neurons.

We report about a setup optimized for simultaneous electrophysiological multi electrode patch clamp and multi photon laser scanning fluorescence microscopic experiments on brain slices.

The microscopic system is based on a modified commercially available confocal scanning laser microscope (CLSM). From a technical and operational point of view, two developments are important:

Firstly, in order to reduce the workload for the experimentalist, who in general is forced to concentrate on controlling the electrophysiological parameters during the recordings, a system of shutters has been installed together with dedicated electronic modules protecting the photo detectors against destructive light levels caused by erroneous opening or closing of microscopic light paths by the experimentalist.

Secondly, the standard detection unit has been improved by installing the photomultiplier tubes (PMT) in a Peltier cooled thermal box shielding the detector from both room temperature and distortions caused by external electromagnetic fields. The electrophysiological system is based on an industrial standard multi patch clamp unit ergonomically arranged around the microscope stage.

The electrophysiological and scanning processes can be time coordinated by standard trigger electronics.
\end{abstract}

Keywords: interneurons, electrophysiology, brain slice, multi photon, scanning laser microscopy, two photon, patch clamp, Peltier cooling, cooled detector

\section{INTRODUCTION}

The patch clamp method ${ }^{1-3}$ in cellular electrophysiology has been a motor for the progress in experimental cell physiology since its first successful application in 1976, and Bert Sakmann and Erwin Neher, who had developed the method, were awarded the Nobel Prize in Medicine and Physiology in 1991 for their seminal work with this method. Following its first publication in $1990^{4}$, MPLSM has developed into a method of choice for high resolution optical microscopy on living biological preparations, both in-vivo ${ }^{5-9}$ and, sometimes combined with the patch clamp method, invitro $^{10-12}$. Properly applied, the photon damage induced onto the specimen can be minimized when employing MPLSM, and cell physiological processes may be observed during hours, and can be time coordinated with simultaneously running electrophysiological measurements. The apparatus described in this paper is designed for experimental studies of dynamic signal processing in the dendrites of neurons in the mammalian brain involving optical recordings with MPLSM

\$ To whom correspondence should be addressed.

$\Xi^{\circ}$ : Centre for Molecular Biology and Neuroscience - University of Oslo, Institute of Basic Medical Sciences, Dept. of Anatomy, P.O. Box 1105 - Blindern, NO-0317 Oslo, Norway, 留: +47 228 51159, 且: +47 228 51499, 吕: p.j.helm@medisin.uio.no, 圈: www.cmbn.no 
time coordinated to electrophysiological recordings with multi-electrode patch clamp techniques ${ }^{13}$.

Most neurons in the brain have an elaborate dendritic tree with a highly complex branching structure, which collects synaptic inputs from other neurons, shapes and integrates the synaptic signals in complex manners, and funnels the resulting signals to the soma. The dendritic branches compartmentalize their primary inputs and may contain a variety of active conductances that markedly modify the synaptic input ${ }^{14}$. Thus, for proper studies of dendritic signal processing, recording techniques are needed that can capture simultaneous activity in different compartments as well as temporal changes and propagation of activity across dendrites.

The setup described here was developed to achieve this through optical recordings of fluorescent dendritic signals, e.g. $\mathrm{Ca}^{2+}$ signals, by means of MPLSM from neurons in brain slices. Slices should be cut such that the major afferent fibers to the neurons to be recorded are intact. Thereby, responses can be evoked by electrical stimulation of the afferents to reveal a possible distinct compartmentalization of inputs from the different types of afferents, patterns of propagation of the evoked activity, sites and conditions for eliciting specific active conductances, and patterns of integration of different types of synaptic input. The setup might also be used for studies of responses to stimulation at different specific points of the dendrite by photolysing caged glutamate through a separate laser beam ${ }^{15,16}$. Moreover, the setup might be used to study synaptic transmission between synaptically connected pairs of neurons in the slice by electrical stimulation of the pre-synaptic neuron and optical recording of the evoked response in the pre-synaptic terminal and in the dendritic site of the post-synaptic neuron ${ }^{17}$.

The optical recordings could be supplemented by electrical whole cell recordings from the soma to determine how different types of dendritic activity are reflected by electrical responses in the soma. Furthermore, this combination of methods could be used to study dendritic effects of back propagation of soma-generated action potentials into the dendrites, for example patterns of back propagation, and location of possible "hot spots" where back propagation activates specific active conductances. The setup has an efficient PMT based system of photo detection for MPLSM which is time coordinated with an industrial multi patch clamp system.

Considerable efforts have been focused on the development of a system to protect the photo detectors from accidental exposition to light sources of a destructive light level. This system is also described in detail in this article.

\section{DESCRIPTION OF THE SYSTEM}

\subsection{The layout of the system}

The system described here is installed on a passively vibration isolated optical table top and assembled around a large frame fixed stage upright microscope. An industrial multi patch clamp unit includes a robust stage, which can be moved at micron resolution laterally by means of two step motors, while focusing is controlled via a third step motor attached to the fine focus mechanism of the microscope. Attached to the stage are four micro manipulators for pipettes individually controlled at micron resolution by sets of three step motors, each providing an independent mechanical degree of freedom. The bath chamber is fixed in the center of the stage.

Both, the microscope and the stage of the multi patch clamp unit are located close to each other, their frames partly covering each other, and screwed to the optical table. However, they are not directly screwed to each other mechanically, preventing inevitable sources of mechanical vibration on the microscope (clicking filter holders, flapper mirrors a. s. o.) from directly transmitting waves potentially destructive for the patches to the stage. The transmission via the optical table top is strongly dampened.

An industrial beam scanning unit, user controlled by standard software via a PC, is attached to the microscope. The beam of a mono frame yttrium vanadate pump laser and titanium sapphire laser (Ti:Sap Laser) including a user friendly, mouse controlled prism group velocity dispersion compensator ${ }^{18}$ (GDC) is directed towards the scanning unit via an Electro Optic Modulator (EOM), which is controlled via the frame active or the pixel clock signal sent from the electronic control unit of the scanner. A beam expander including a spatial filter cleans the modulated beam and expands it to fill the scanning mirrors, which sweep it, while scanning, over the specimen.

The microscope is also equipped with a fiber coupled $\mathrm{Hg}$ arc lamp, providing uniform illumination of the entire field of view for conventional fluorescence observation, as well as a 12V/ 100W halogen lamp, DIC equipment, IR filters and a camera system for IR DIC microscopy ${ }^{19}$.

The system will shortly be equipped with a second laser system similar to the one already installed. With two active lasers, it will, e.g., be possible to optically release caged compounds by means of a two photon process ${ }^{20}$, while simultaneously at least one other dye substance can be excited for fluorescence observation using the first laser.

\footnotetext{
$\S$ The used components are listed in an Appendix at the end of this article.
} 


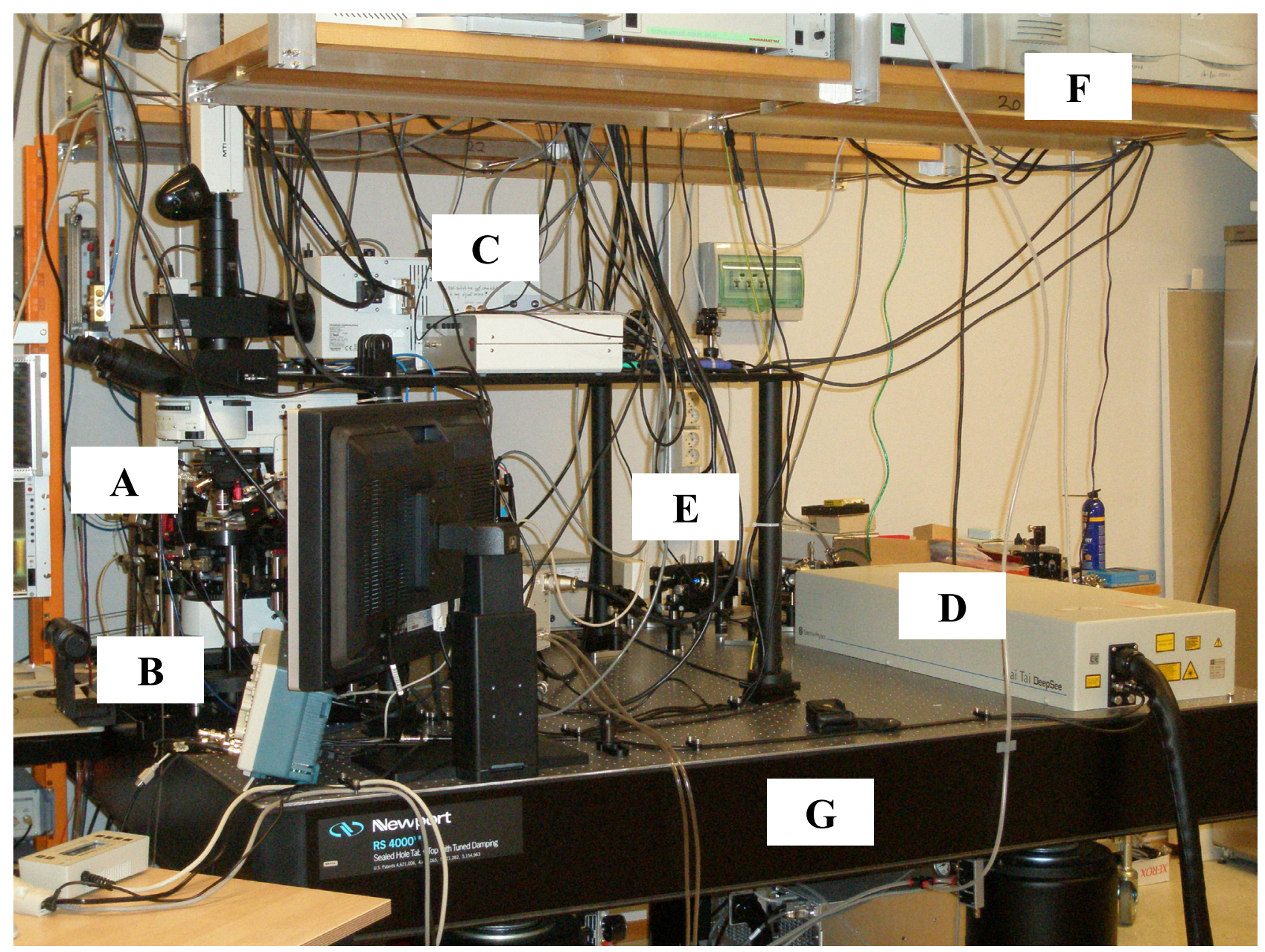

Fig. 1: Setup overview

In this picture, the entire setup is imaged.

The letters designate the following components:

A: microscope,

B: $\quad$ stage and micro manipulators,

C. scanner,

D: laser,

E: beam ray path including EOM and beam expander with spatial filter,

F: shelves for electronic components,

$\mathrm{G}$ optical tabletop on vibration isolators.

\subsection{The potential sources of destructive light levels for the detectors}

The microscopic system, which is the platform for the establishment of the preparation in the objective focal region, proper patching of cells and the detection of fluorescent light as optical signal of cell physiological phenomena, has to serve three purposes, namely classical differential interference contrast (DIC) visual wide field microscopy, infrared DIC camera microscopy, and MPLSM. For the latter, efficient photon detection demands that the detection units, in casu. photomultiplier tubes (PMT), be placed as close as possible above and below the source of the fluorescent light, i.e. the specimen. Unavoidably, the highly sensitive PMTs are, hence, positioned in close neighborhood to strong light sources. Before switching on the high voltage (HV) to the detectors, light from all sources of the setup besides the Ti:Sap laser must be switched off, and so has to be the room light.

Patch clamp experiments demand a sustained and high level of concentration. The experimentalist permanently has to actively control numerous parameters in order to keep the actual brain slice in good shape, she or he at the same time has 
to supervise the electrophysiological recordings and, in addition, the scanning process of the MPLSM. Especially younger and less experienced experimentalists will be stressed.

There is, hence, a permanent danger that the experimentalist accidentally opens light paths on the microscopic platform or switches on light sources that would expose the sensitive and expensive MPLSM photo detectors to destructive levels of light.
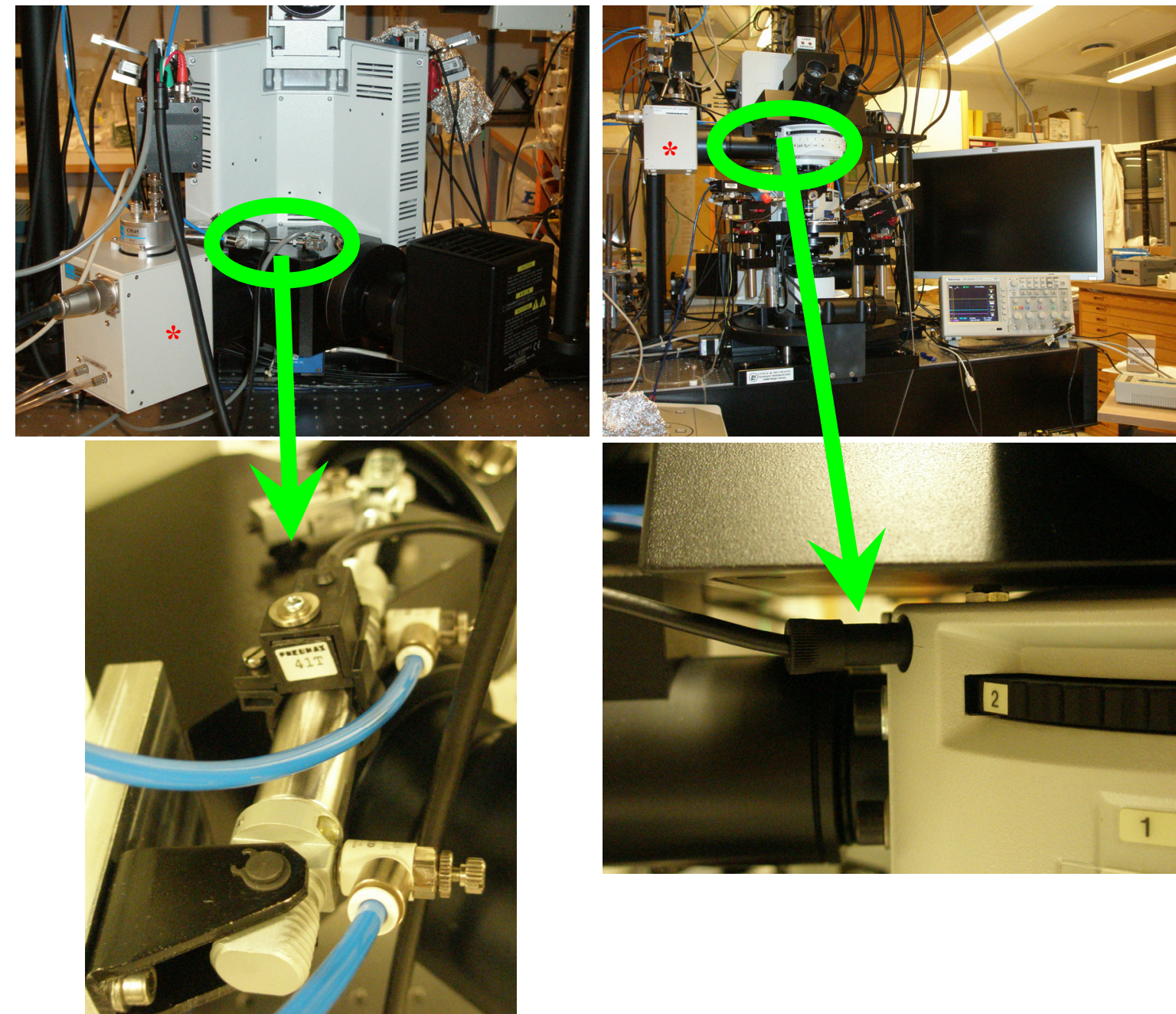

Fig. 2: The attachment of the PMT units, mounted in cooling boxes (see “*” - symbols)

On the left hand side, the detector house and the $12 \mathrm{~V} / 100 \mathrm{~W}$ halogen lamp can be seen, both attached to a cube including a pneumatically remote controlled flapper mirror with position detector.

On the right hand side, a detector house is attached to the housing of the epi-illuminator, in direct vicinity to the filter revolver used for placing filters, either directing the light by an $\mathrm{Hg}$ arc lamp to the specimen or directing the MPLSM fluorescent light to the detector. The green arrows indicate the position detectors (see text).

The two PMTs of the MPLSM, set to register light from the specimen via the objective and via the condenser, see Fig. 2, are placed in cooling boxes. By means of Peltier elements, the tubes are cooled to approx. $-30^{\circ} \mathrm{C}$. One of the units is attached laterally to the epi-illuminator, the other is attached to a flapper mirror mounted to the rear lower end of the microscope. This flapper mirror either directs the light of the $12 \mathrm{~V} / 100 \mathrm{~W}$ halogen lamp into the microscope or the signal from the specimen towards the PMT.

Signal light, in both cases, is focused onto the entrance window of the PMT be means of a suitable lens. Both PMTs are protected against stray light from the laser by means of optical filters suppressing light in the wavelength range of the lasers at a rate of $10^{-8}$ at least. 


\subsection{System of protective shutters, sensors, and control electronics}

to / from tube lens, binocular, laser, scanner, and additional wide field camera

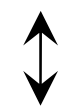

Arc lamp for epi-illumination wide field fluorescence microscopy

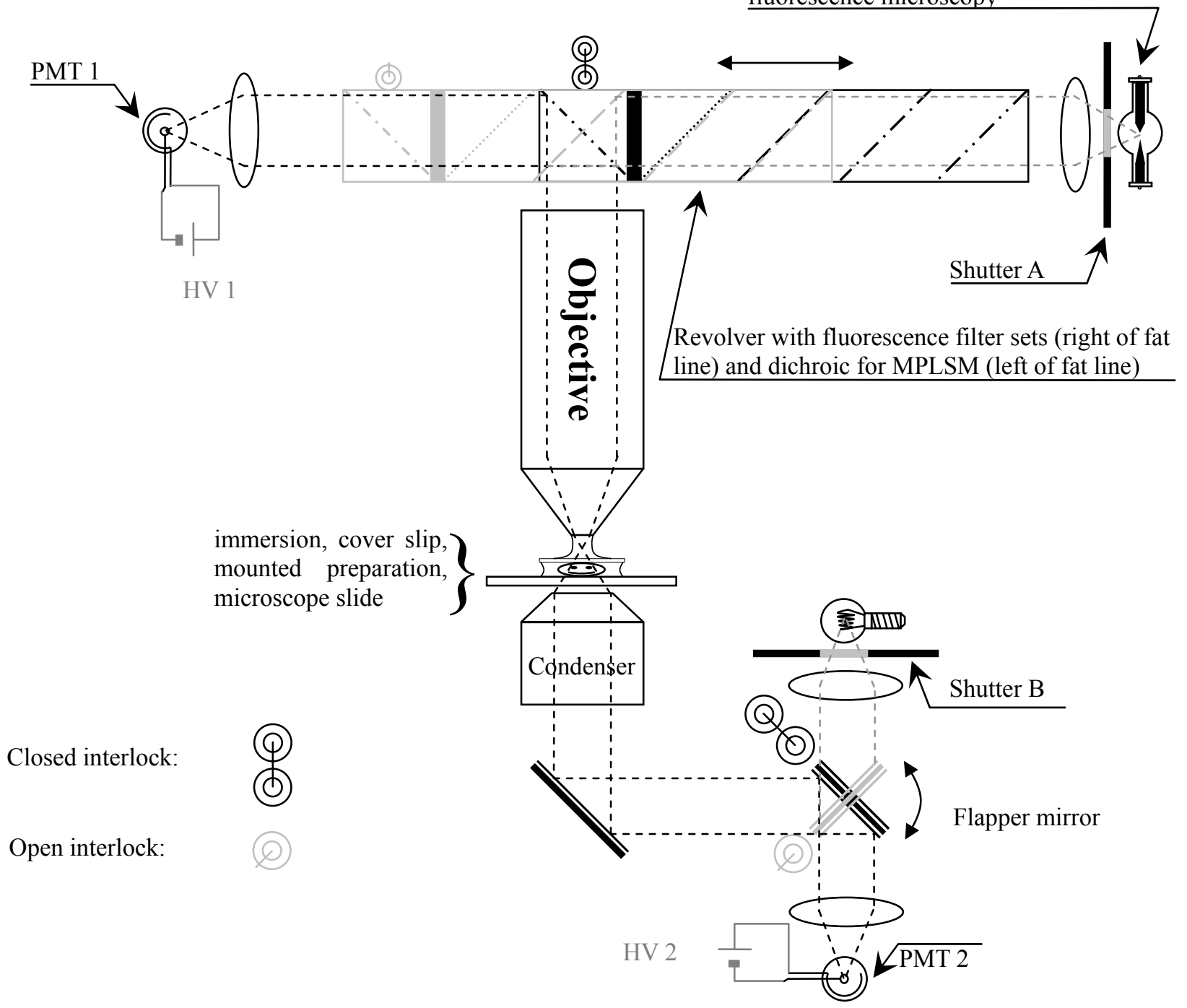

Fig. 3 : Protective System

This figure shows the main elements, which establish the protective system for the PMTs, within the frame of the microscopic system. Grey color indicates alternative setting resp. positioning or devices that have to be switched in order to function or provide system safety. If HV1 or HV2 are being activated, the shutters are automatically being closed so that no light from the light sources can expose the photo detectors from destructive light levels. Also, the room light is automatically being switched off.

Likewise, if one of the two interlocks or both erroneously is resp. are opened, irrespectively of whether one of the HVs is switched on, both shutters are automatically closed.

The closure of the shutters takes milliseconds. They cannot be re-opened by the user before the HVs have been switched off, the room light has been switched off and the interlocks have been closed by bringing the revolver with the fluorescence filters and the flapper mirror to their resp. correct positions. 
In order to avoid the scenario described in 2.2, a protective system has been developed consisting of industrially produced electronic shutters, position detectors for the aforementioned flapper mirror and epi-illuminator filter revolver as well as an electronic unit constructed and built in the local electronics laboratory providing trigger, gate, and reset signals to the shutter controllers, see Fig. 3.

The "dangerous" situations will be the following:

1)

The system is being used for wide field microscopy.

The 12V / 100W halogen lamp OR the Hg arc lamp OR the room light are switched ON.

The flapper mirror AND the fluorescence filter revolver (see Fig. 2) are in the correct positions for wide field microscopy transmitted light OR epi-fluorescence, HV1 AND HV2 are OFF.

a)

Both shutters are open.

b)

The gate signal controlling the trigger input to the shutter controllers is HIGH.

c)

If, erroneously, HV1 OR HV2 are switched ON, both shutter controllers immediately receive a trigger signal to their gated input, thus closing the shutters at once. $100 \mathrm{msec}$ later, the gate signal for the trigger signal is set to LOW, thus inhibiting any further trigger signals, which erroneously might be sent to re-open the shutters. The Gate signal has to be manually reset to HIGH. Before this can be done, both HV1 AND HV2 have to be switched OFF:

2)

Situation as in 1)

a)

see 1).

b)

see 1).

c)

If, erroneously, the flapper mirror OR the fluorescence filter revolver are moved from their positions, both shutter controllers immediately receive a trigger signal to their gated input, thus closing the shutters at once. $100 \mathrm{msec}$ later, the gate signal for the trigger signal is set to LOW, thus inhibiting any further trigger signals, which erroneously might be sent to re-open the shutters.

Thus, even in case of de-activated HV, the detectors are protected from being hit by strong light, avoiding increased noise afterwards. Wide field microscopy is thus, impossible, if the flapper mirror OR the fluorescence filter revolver are in un-suitable positions.

3)

The system is being used for MPLSM.

Both shutters are closed AND the room light is OFF AND (HV1 OR HV2 are ON))

In this case, the gate signal controlling the trigger input is LOW.

In this situation, it is impossible to open any of the shutters or switch ON the room light.

First, both HV1 AND HV2 have to be switched OFF, the flapper mirror and the epi-fluorescence filter revolver be brought to appropriate wide field microscopy positions, and the Gate signal switched to HIGH by pressing a button. The gate signal cannot be set to HIGH unless both HV1 AND HV2 are off.

\section{RESULT}

The functionality of the described system described has been tested successfully. The setup will be a useful tool for the planned experiments. 


\section{ACKNOWLEDGEMENTS}

The multi photon laser scan facilities and auxiliary infrastructure described in this publication were financed by the Norwegian Research Council.

The help of the engineers of the local Mechanical Workshop, Ali Fakheri, Thomas Ingebricson, Torgeir Kvernstuen, Knut A. Sørensen Rekdahl, Rolf Bakke, and Reidar Larsen as well as of the head of the Electronics Laboratory, Bjarne Authén, is gratefully acknowledged. Jon I. Wedervang of the local IT department assisted effectively with the establishment of a network for all the PCs needed for the setup.

\section{REFERENCES}

[1] Neher, E. and Sakmann, B., "Single-channel currents recorded from membrane of denervated frog muscle fibers," Nature 260:799-802 (1976)

[2] Neher, E. and Sakmann, B., "Noise analysis of drug induced voltage clamp currents in denervated frog muscle fibres," J. Physiol. 258:705-729 (1976)

${ }^{[3]}$ Neher, E., Sakmann, B. and Steinbach, J.H., "The Extracellular Patch Clamp: A Method for Resolving Currents through Individual Open Channels in Biological Membranes," Pflügers Arch. 375:219-228 (1978)

[4] Denk, W., Strickler, J.H. and Webb, W.W., "Two-photon laser scanning fluorescence microscopy," Science 248:73-76 (1990)

[5] Trachtenberg, J.T., Chen, B.E., Knott, G.W., Feng, G., Sanes, J.R., Welker, E. and Svoboda, K., "Long-term in vivo imaging of experience-dependent synaptic plasticity in adult cortex," Nature 420:788-794 (2002)

[6] Grutzendler, J., Kasthuri, N. and Gan, W.-B., "Long-term dendritic spine stability in the adult cortex," Nature 420:812-816 (2002)

[7] Ottersen, O.P. and Helm, P.J., "How hardwired is the brain?," Nature 420:751-752 (2002)

${ }^{[8]}$ Nase, G., Helm, P.J. and Ottersen, O.P., "A multiphoton laser scanning microscope setup for transcranial in vivo brain imaging on mice," Review of Scientific Instruments 76:123702-1-123702-5 (2005)

[9] Nase, G., Helm, P.J. and Ottersen, O.P., "Water entry into astrocytes during brain edema formation," Glia 56(8):895$902(2008)$

${ }^{[10]}$ Nevian, T. and Sakmann, B., "Single spine $\mathrm{Ca}^{2+}$ signals evoked by coincident EPSPs and backpropagating action potentials in spiny stellate cells of layer 4 in the juvenile rat somatosensory barrel cortex," The Journal of Neuroscience 24(7):1689-1699 (2004)

${ }^{[11]}$ Mulligan, S.J. and MacVicar, B.A., "Calcium transients in astrocyte endfeet cause cerebrovascular constrictions," Nature 431:195-199 (2004)

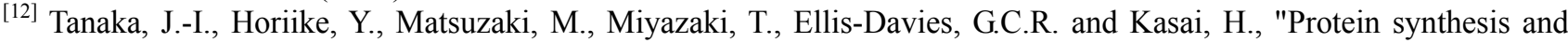
neurotrophin-dependent structural plasticity of single dendritic spines," Science. 319:1683-7 (2008)

${ }^{[13]}$ Helm, P.J., "A microscopic setup for combined, and time-coordinated electrophysiological and confocal fluorescence microscopic experiments on neurons in living brain slices," Rev. Sci. Instr. 67(2):530-534 (1996)

${ }^{[14]}$ Losonczy, A., Makara J.K. and Magee, J.C., "Compartmentalized dendritic plasticity and input feature storage in neurons," Nature 452:436-442 (2008)

${ }^{[15]}$ Major, G., Polsky, A., Denk, W., Schiller, J. and Tank, D.W., "Spatiotemporally graded NMDA spike/plateau potentials in basal dendrites of neocortical pyramidal neurons," J. Neurophysiol. 99:2584-2601 (2008)

${ }^{[16]}$ Nikolenko, V., Poskanzer, K.E. and Yuste, R., "Two-photon photostimulation and imaging of neural circuits," Nature Methods 4:943-950 (2007)

${ }^{[17]}$ Kaiser, K.M.M., Lübke, J., Zilberter, Y. and Sakmann, B., "Postsynaptic calcium influx at single synaptic contacts between pyramidal neurons and bitufted interneurons in layer $2 / 3$ of rat neocortex is enhanced by backpropagating action potentials," J Neurosci 24:1319-1329 (2004)

${ }^{[18]}$ Fork, R.L., Martinez, O.E. and Gordon, J.P., "Negative dispersion using pairs of prisms," Opt. Lett. 9(5):150-152 (1984)

${ }^{[19]}$ Dodt, H.-U. and Zieglgänsberger, W., "Visualizing unstained neurons in living brain slices by Infrared DICvideomicroscopy," Brain Res. 537:333-336 (1990)

${ }^{[20]}$ Losonczy, A. and Magee, J.C., "Integrative Properties of Radial Oblique Dendrites in Hippocampal CA1 Pyramidal Neurons," Neuron 50:291-307 (2006) 


\begin{abstract}
APPENDIX
The setup is installed on a model "M-RS4000-56-8" optical table top, $1500 \mathrm{~mm} \times 1800 \mathrm{~mm} \times 305 \mathrm{~mm}$ mounted on four model "I-2000-423.5" isolators. Both are by Newport Corp. Irvine, CA, USA.

The lasers are a model "Mai Tai Deep See", including a Nd:YVO 4 pump laser and a Ti:Sap laser as well as a remote controlled prism GDC, by Spectra Physics Laser Division, Mountain View, CA, USA, and a model "Chameleon" including a Nd:YVO 4 pump laser and a Ti:Sap laser, by Coherent Inc., Santa Clara, CA. USA.

The opto-mechanical components are mostly by Newport, but include some home made components built in the local mechanical workshop and anodized at Eloksal AS, Horten, Norway.

The EOM is a model "LM0202 PW 5 IR" four crystal K*DP modulator controlled by a model LIV20 pulse amplifier, both by LINOS Photonics GmbH \& Co. KG, München FRG.

The beam expander and spatial filter are assembled from a model "MRF56" spatial filter, a model "MFB 1.050" pinhole with $50 \mu \mathrm{m}$ in diameter, and model "OFS 8.3.20" and "OFS 8.3.06" Fraunhofer Achromats, all by Bernhard Halle Nachfl. Optische Werkstätten, Berlin, FRG, as well as a model "LP-1A" multi axis lens positioner including a model "LP-LH0" semi fabricated lens holder, all by Newport Corp., Irvine, CA, USA.

The microscope is a model "BX51WI" including a model "LUMPlanFL N 40×/0.8 W" and a model "MPlan N $5 \times / 0.10$ " objective by Olympus, Tokyo, Japan. The microscope is equipped for epi-fluorescence and transmitted light IR-DIC microscopy. The standard objective holder "WI-SRE2" has been replaced with a model "FV10-SRE" holder including a WI-DICTHRA DIC prism. Other than in the "WI-SRE2", in the "FV10-SRE" the DIC prism is placed in a slider and can be easily be put into or removed from the microscope ray path in order to not distort laser scanning microscopy.
\end{abstract}

Attached to the microscope is a model "FV300" scanning unit, also by Olympus.

The two PMTs are model "R7683" (Hamamatsu Photonics KK, Hamamatsu City, Japan). The "R7683" is a high efficiency OEM version of the model "R3896" PMT. The PMTs have been mounted in model "C9143" cooling boxes equipped with model "C9145" DP-type sockets (all by Hamamatsu) including Cockroft-Walton style high DC voltage sources. Hamamatsu specifies the improvement of the $\mathrm{S} / \mathrm{N}$ ratio of the detected light attained by the cooling and electromagnetic isolation of the PMT as a factor of 18.2 as compared to the signal detected by the unshielded and uncooled detector. This figure cannot be checked by the user since Hamamatsu does not provide the software with which it would be possible to operate the PMT in the cooling box, i.e. with the same socket and HV-source, at room temperature. It should, however, be mentioned that earlier experiments done with a PMT not cooled and not shielded were not successful since the weak fluorescent signal was drowned in noise in spite of the fact that the PMT use during these earlier experiments, a model "R6357" by Hamamatsu, even had a slightly better quantum efficiency than the type used during the measurements described in this publication. Cooling and shielding the PMT thus appear to be essential for these experiments. The PMT signal is read out via model "C7319" amplifiers (Hamamatsu) to the 12 bit AD converter of the "FV300".

The cooling water circuit to the C9143 boxes is pumped by a UPS protected model "DGS.99PPPT2NN0M103" stainless steel gear pump by Tuthill Pump Group, Concord, CA, USA, coupled to a 3-phase electro motor and HZ transformer supplied by Fuglesangs AS, Oslo, Norway.

The C9143 housings are attached to the microscope by home made parts (see Fig. 2) and system modifications. In order to direct the light to the PMTs, a model "IX2-MFB-SP-R" reflector, placed in the fluorescence filter revolver of the epiilluminator, resp. a model "Lampenhausumschaltung für Olympussyteme" flapper mirror attached to the rear transmitted light ray path connector of the microscope, all by Olympus, were used. The dichroic filter installed in the IX2-MFB-SP$\mathrm{R}$ is a model "670dcxru" filter by Chroma Technology Corp., Rockingham, VT, USA. Model "CKX075AR.14" cylinder lenses by Newport are used to focus the fluorescent light (resp. possibly SHG light in case of the transmitted light detector) onto the active surfaces of the PMTs. Model "ET670sp-2p" filters by Chroma Technology Corp., Rockingham, VT, USA, protect the PMTs at an OD of at least 8 from stray light in the wavelength range of the light emitted by the lasers.

The shutters used to protect the PMTs from erroneously switched on light sources were model "VS35S2ZM1-24" shutters controlled by model "Uniblitz VCM-D1" controllers, both by Vincent Associates, Rochester, NY, USA. A third shutter, used to block the laser beam from the "Mai Tai Deep See" is model "LS6ZM2"shutter, also this one controlled by a model "Uniblitz VCM-D1" controller, all by Vincent Associates.

The microscope stage and the micromanipulators are by Luigs \& Neumann Feinmechanik und Elektrotechnik GmbH, Ratingen, FRG, and consist of a model "380FM" workstation including two SM-5 / SM-5 III units with altogether four model "LN Mini 25" micromanipulator units with three degrees of freedom, each. Moreover, a "Badcontroller V" unit is used on the system. 